https://helda.helsinki.fi

\title{
Is Open Hardware Worthwhile? Learning from Thales' Experience with RISC-V
}

\section{Legenvre, Herve}

2020-07-03

Legenvre , H , Kauttu , P , Bos , M \& Khawand , R 2020 , ' Is Open Hardware Worthwhile? Learning from Thales' Experience with RISC-V ', Research Technology Management, vol. 63 , no. 4 , pp. $44-53$. https://doi.org/10.1080/08956308.2020.1762445

http://hdl.handle.net/10138/338660

https://doi.org/10.1080/08956308.2020.1762445

cc_by_nc

acceptedVersion

Downloaded from Helda, University of Helsinki institutional repository.

This is an electronic reprint of the original article.

This reprint may differ from the original in pagination and typographic detail.

Please cite the original version. 


\section{Is Open Hardware Worthwhile?:}

\section{Learning from Thales' Experience with RISC-V}

Hervé Legenvre, Pietari Kauttu, Martin Bos, and Roger Khawand

\section{Bios below}

Hervé Legenvre is a professor at The European Institute of Purchasing Management (EIPM) in France. He leads EIPM's research activities and is an assessor for the EIPMPeter Kraljic Awards. His research interests include innovation, ecosystems, the Internet of Things, and open source initiatives. hlegenvre@eipm.org

Pietari Kauttu is vice-president of Consensus Capital LLC and a senior industrial collaboration advisor at The Helsinki Institute of Physics. His background is in R\&D and innovation financing. He has conducted research on novel technology collaboration mechanisms, including open source hardware, at TUNI/CERN. Pietari.Kauttu@cern.ch

Martin Bos is a senior procurement manager at Thales, working in software engineering and systems subcontracting. Since 2008, he has played a key role in Thales' advanced research of open source software in an OEM business context. He has extensive experience in high-impact transformation projects focused on driving business value through technology, strategic innovation, and competitiveness initiatives. martin.bos@nl.thalesgroup.com

Roger Khawand is procurement director at Thales, where he focuses on the railway, defense, and aerospace domains. Using his strong business acumen and breakthrough ideas in procurement strategy, he has shepherded Thales successfully through the implementation of commercial off-the-shelf hardware and software in military projects, using innovative industry and process optimization. roger.khawand@thalesgroup.com

Overview: In this article, we frame the concept of a hardware-rich open source ecosystem (H-ROSE) that generates software and hardware components. In an H-ROSE, the designs of some components are accessible under open source licenses, while other component designs remain proprietary. We describe seven adoption factors used by the multinational French firm Thales to assess the efficacy of RISC-V to design processors. Other companies can use these adoption factors to explore whether an open hardware initiative supported by an H-ROSE is worthwhile.

Keywords: Open source, Adoption factors, Ecosystems, RISC-V, H-ROSE 
Until the mid 2010s general-purpose processors progressed continuously and could support computing and mobile applications. Currently, they can no longer support all the emerging needs related to the consumer Internet of Things (IoT), the industrial IoT, and the automotive sector. Development costs for processors are also rising quickly at a time when many companies urgently need low-cost, custom-purpose processors. Companies are turning to RISC-V, an open source solution to create such customer-purpose processors, and they join the RISC-V ecosystem to achieve their objectives.

The term ecosystem is increasingly associated with innovation. An ecosystem comprises heterogeneous but interdependent organizations that combine complementary products and/or services to innovate and deliver a value proposition (Adner 2017; Jacobides, Cennamo, and Gower 2018). For example, companies, organizations, and individuals supporting open source software projects form an ecosystem. In the last 10 years, some large open source ecosystems have moved beyond software to include hardware components. In these ecosystems, participating companies work, for instance, on microprocessors, data centers, telecom infrastructure, autonomous vehicles, and the IoT. We call an ecosystem that includes hardware components a hardware-rich open source ecosystem (H-ROSE).

The RISC-V ecosystem is one example of an H-ROSE. RISC-V provides an open instruction set architecture (ISA), which serves as the interface between software and hardware within a processor. RISC-V supports the development of open processors. Currently, the main providers of ISA are Arm, the British multinational semiconductor and software design company owned by Softbank, and Intel, the American multinational technology company. The RISC-V Foundation, established in 2015, has more than 400 member organizations that use the RISC-V ISA. RISC-V is prevalent in academia and, increasingly, large commercial firms use it. For example, Western Digital uses RISC-V for storage applications, NVIDIA uses it for graphic processors, and Xiaomi's Huami brand uses it in wearables. Samsung and Alibaba have announced RISC-V projects for 5G, artificial intelligence (AI), the Internet of Things, and autonomous vehicle applications.

As more and more companies consider joining an H-ROSE like RISC-V, they need guidance on for their decision-making. We describe seven adoption factors firms can usethey emerged from an in-depth case study in which we explored how the multinational French firm Thales has adopted RISC-V to design its processors. Thales is active in the aerospace, defense, transportation, and security markets. We tested the adoption factors with other RISC-V ecosystem participants, from the CERN open hardware community, and from other H-ROSEs. We determined that RISC-V is a viable solution to create custom-purpose processors.

\section{Hardware-Rich Open Source Ecosystem}

Open source involves making bodies of original materials such as code for software or design files for hardware publicly available for others to use (Lerner and Tirole 2005). Open source originated with the free software movement in the 1980s that aimed to make 
the source code of software availaible so it could be freely used, copied, distributed, studied, and modified. According to Pénin (2011), open source initiatives also exist in the clothing and music industries as well as in the biological sciences. Open source hardware developed first within scientific and technical circles outside of mainstream commercial projects (Kauttu and Murillo 2017). Several large open source ecosystems appeared in the commercial realm in the 2010s (Bonvoisin et al. 2017). We coined the term hardwarerich open source ecosystem (H-ROSE) to describe an ecosystem that includes software and hardware components. A subset of the components is accessible under open source licenses. We highlight five H-ROSE initiatives, though more may exist (Table 1). To characterize an H-ROSE further, we compare it to standardization bodies, traditional open source software inititiatives, and previous open hardware projects.

\section{-- Table 1 near here - -}

Our use of the term ecosystem aligns with the literature on technology standards (Naranayan and Chen 2012) and with the recent development of the ecosystem concept (Jacobides, Cennamo, and Gower 2018). An H-ROSE develops standards but differs from traditional standardization bodies or R\&D consortia in several ways. First, an HROSE includes not only firms that contribute to the standard, but also firms that provide additional offerings such as services, software tools, equipment, or manufacturing capabilities needed to form a full supply chain and deliver final applications. Second, an H-ROSE results from market-shaping strategies adopted by one or more firms. Following Gambardella and von Hippel's (2019) study of the Open Compute Project (OCP), such market-shaping strategies are either upstream or downstream. An upstream strategy aims to create an alternative to a supplier's proprietary design - it can replace a dominant supplier with a new supply network where no firms benefit from a lock-in situation. The Telecom Infra project (TIP), RISC-V, and OCP are examples of H-ROSEs created to implement upstream strategies. By contrast, a downstream strategy aims to carve a space for a new offering in a new market. APPOLO AUTO and LoRa (Long Range) are HROSEs created to implement dowstream strategies. Finally, open source helps strategically lower entry barriers for providers of complementary offerings in an $\mathrm{H}$ ROSE, so the ecosystem can grow rapidly.

Compared to open source software solutions, an H-ROSE faces specific challenges in terms of quality, validation, and supply chain. Quality issues can be significant - for instance, processors with defects in the code cannot be repaired. Testing, validation, and certification costs can be high-for example, testing autonomous cars requires a large amount of data. Finally, an HROSE needs to develop dedicated supply chains with multiple actors capable of delivering specific volumes of custom applications. In downstream strategies, open source supply chains compete with well-established integrators offering turn-key solutions. In upstream strategies, these supply chains compete with alternative solutions in a context of uncertainty.

H-ROSEs differ from past open hardware initiatives: they orchestrate collaborative efforts across a large group of multinational companies with extensive innovation capabilities. Scientists and independent inventors initiated the development of open hardware. One example is scientists in CERN that pioneered the development of 
affordable open source scientific equipment (Kauttu and Murillo 2017). A second example is a community of users and independent inventors that created open source medical devices and protocols to treat diabetes because healthcare companies were too slow to respond to user needs. A third example is Local Motors, a small innovative company that open sourced its autonomous fleet vehicles and support applications in niche markets. In contrast with such open hardware initiatives, an H-ROSE brings together hundreds of multinationals with extensive innovation capabilities. An H-ROSE aspires to change how some industries operate whereas prior open hardware initiatives dealt with market niches.

Since the H-ROSE is a new phenomenon, questions remain: What factors influence the adoption of an H-ROSE? How is an H-ROSE governed? To what extent can an H-ROSE be successful over time? In this article, we focus on what factors influence the adoption of an H-ROSE. We endeavor to answer this question using a case study centered on the RISC$\mathrm{V}$ ecosystem.

\section{The Case Study}

Thales Group is a French multinational company active in the aerospace, defense, transportation, and security markets. It has a central Research and Technology team that supports the company's different business units with their technology development. In early 2018, Thales appointed a team of two experienced purchasing managers to work with the Research and Technology team to assess the benefits and risks of joining the RISC-V ecosystem. The overall project comprised workshops and discussions with representatives from some of Thales' business units, from the RISC-V Foundation, and from potential suppliers and organizations active in the RISC-V ecosystem that contribute to the design and manufacturing of processors based on RISC-V.

\section{Methodology}

To understand the factors that influence the adoption of an H-ROSE, we built on the theory of innovation diffusion (Wejnert 2002). Following Wejnert (2002), we looked first at the characteristics of the innovation, including the benefits and costs associated with its adoption. Second, we considered the industry characteristics of the actors adopting the innovation. We did not consider broader environmental factors, Wejnert's (2002) third set of factors, because they are geared toward consumer markets. However, where appropriate, we discussed some environmental factors such as the current trade dispute between the United States and China. The development of the seven adoption factors builds on a case study approach that we found suited our investigation of a real-world contemporary phenomenon. Throughout our work we followed the guidelines provided by Tsoukas (2009) and Mariotto, Zanni, and De Moraes (2011): they suggested reflecting on what the characteristics are and what occurs within the case before generalizing the findings.

Our findings emerged as we progressed through multiple steps. Initially, we gathered empirical evidence that includes more than 1,000 pages of internal documents, presentations, and email correspondence that reflect the views of Thales' key decision makers. Then we conducted semi-structured interviews of about 60 minutes each with seven Thales 
managers, directors, and executives (Table 2). Following Eisenhardt (1989), we finalized data collection when no significant additional insights emerged from the interviews. We inductively coded the empirical evidence to develop a first set of factors and subfactors. We based the first level of coding on the exact words used by interviewees or found in the internal documents Thales provided. The first level of coding yielded 46 subfactors, which we grouped into 14 main factors that two of our researchers subsequently titled.

\section{- - Table 2 near here - -}

We then performed new semi-structured interviews with three of the Thales representatives to refine the description of the factors. This led us to a list of 17 adoption advantages and 9 limitations that we aggregated into 7 main adoption factors. During this process we simplified, grouped, or refined some of the initial 46 subfactors.

We tested the adoption factors with three representatives from the RISC-V ecosystem to assess the impact of industry characteristics. We then compared our findings with the literature on open source software adoption and tested our factors through semi-structured interviews with two representatives from the CERN open hardware community and with two representatives from other H-ROSEs. Although we made minimal changes to the wording of the factors, it helped us realize that the factors' importance can vary significantly across different applications, industries, or H-ROSEs. Concerns related to cybersecurity are one example of such variance. Our comprehensive process gave us confidence that firms can use our adoption factors to make decisions regarding H-ROSEs. The factors are relevant and provide a robust way for firms to assess whether or not they should join an H-ROSE.

\section{Results}

We identified seven adoption factors:

1. Total cost optimization, including the cost of accessing innovation

2. Flexible and rapid design process

3. Stability and modularity

4. The white box approach

5. Possibility to select suppliers outside the dominant players

6. Permissive license agreements

7. A growing and active ecosystem

We describe each adoption factor's advantages and limitations (Table 3) and relevance in the case of Thales' adoption of RISC-V. We also outline some lessons learned from the Thales experience that firms can apply. 


\section{Factor 1: Total Cost Optimization, Including the Cost of Accessing Innovation}

The total cost of a new technology is an important adoption factor. Open source licenses lower intellectual property costs and help reduce design costs through the reuse of hardware or software building blocks. When a large group of firms collaborate within an H-ROSE they can pool their creativity and reduce the resources needed to innovate. The cost impact varies across different types of applications; the costs might be positive for some and negative for others. For instance, design costs impact the total cost of low volume and high volume products differently.

For Thales' adoption of RISC-V, this factor proved especially relevant. Given the company's focus on custom-purpose processors, open source solutions offer lower royalty fees and simplified access to technology compared to proprietary solutions. Because Thales Group is active in the aerospace, defense, transportation, and security industries, it has specific needs and low production volumes compared to the mobile phone and automotive industries. Since it has specific needs, Thales can use RISC-V to reduce design costs. One Thales interviewee commented, "Our industry is charaterised by very low volumes of production, so a decrease in terms of IP cost has far more impact for us than for other industries."

Companies and organisations belonging to the RISC-V Foundation provide RISC-V extensions, design tools, and sometimes full processors as open source or easily accessible IP through online configurators. Two open source examples are the NVIDIA Deep Learning Accelerator and an Alibaba processor tailored for 5G, AI, and the IoT (Peng 2019). Online configurators and design services for processors allow companies to to create custom-purpose processors that reuse existing building blocks either available as open source or as zero-cost IP before the production stage (Dubois 2017). This approach reduces the need for internal development skills and makes RISC-V a cost-effective platform for custom-purpose processors. One Thales interviewee said, "In Thales, we are used to developing our own hardware for high-level security applications. This is costly and we might not be state of the art; so, using an open hardware solution is a way to have access to all information we need to ensure security without the constraints of internal developments."

As more extensions, design tools, and processors are open sourced, and multiple partnerships continue to emerge across RISC-V ecosystems, the costs of accessing innovation will decline. For general-purpose processors, RISC-V does not offer distinctive cost advantages compared to an in-house design or an outsourced design. When assessing the opportunity to join an H-ROSE, companies need to consider the total cost impacts of making the change to an H-ROSE on different applications. Performing a multi-application analysis can then be used to develop a progressive development roadmap. Cost calculations should also include different options in terms of insourcing or outsourcing of design or implementation activities. 


\section{Factor 2: Flexible and Rapid Design Process}

The development of an H-ROSE can reduce administrative steps and barriers for new development. Administrative steps such as signing non-disclosure agreements (NDAs) and agreeing on intellectual property rights upfront are often lengthy processes (Glandien 2019). An H-ROSE also provides rapid design and prototyping capabilities thanks to the contributions of companies and organizations across the ecosystem. Similar to factor 1 , the impact on time varies across different applications.

In the past, it took Thales up to 18 months to sign an NDA with a supplier offering an instruction set architecture. This agreement did not cover other Thales suppliers that needed access to the code. Thales' adoption of RISC-V reduced the time needed to design custom-purpose processors thanks to online configurators and the reuse of existing building blocks. For Thales, RISC-V offers greater flexibility and speed to create processors that meet the company's specific needs compared to alternative solutions such as what Arm offers. Since the RISC-V ecosystem provides rapid design capabilities, Arm has changed some of its policies. In 2019, Arm began to allow custom instructions and simplified access to its intellectual property which, when combined with its design kits that speed up the design procedure for processors, will help the company maintain a solid market position.

When assessing whether to join an H-ROSE, Thales' experience shows that companies need to create an inventory of their needs as well as the design and prototyping solutions available within an H-ROSE. Companies must consider the various applications they need so they can make an informed decision about the best option. The Thales experience also shows that companies must consider broad market dynamics - the benefits an $\mathrm{H}$ ROSE offers are relative to what competitors do and how they respond to market changes.

\section{Factor 3: Stability and Modularity}

The third adoption factor covers the stability and modularity of the open source solutions an H-ROSE offers. Stability includes issues regarding quality, reliability, testing, and certification. Michael Cave, senior director of strategic technology at SiFive, a RISC-V solutions provider, says this modular approach is an advantage: "With RISC-V, when you create an integrated circuit, you do exactly what you need," which means that it helps to minimize power consumption, streamline bill of material costs, and optimize board space (McKeefry 2019). The modular architecture is a great asset of RISC-V even if the RISCV ISA is not technically superior to proprietary solutions.

When Thales considered adopting RISC-V, the cross-functional team tasked with overseeing the decision-making process identified several promising positive outcomes. First, Thales needed an ISA without stability and quality issues. To ensure stability, the RISCV ISA code and extensions are frozen, and the RISC-V Foundation actively promotes the use of a single repository. Some RISC-V ecosystem members have released compliance verification tools, and new developments are underway to support broader verification needs (Tusinschi 2019). As a result, system implementations can be conducted safely 
without jeopardizing investments. Second, since RISC-V offers a simple and modular design, customization is possible by using extensions and design tools. RISC-V has fewer than 50 core instructions (Nervos 2019), while a proprietary solution may have thousands. Having fewer lines of codes is a significant benefit for Thales: since some of its applications are integrated in satellites, it is vital to have very few lines of code to reduce power consumption. For a space application, RISC-V enables developers to select only the functionalities they need and build on top of the core instructions for custom developments.

When deciding whether to join an H-ROSE, companies must assess whether central contributors in the ecosystem can and will address stability and quality issues. By doing so, they can ascertain the ecosystem's long-term success and whether joining it makes good business sense.

\section{Factor 4: The White Box Approach}

Since an open source approach provides full access to the code and design files, it creates a "white box" that allows anyone to fully inspect the software and hardware. A white box approach can help address safety and security concerns and help prevent potential IP infringement. However, having access to the code is only of value if the code is properly and systematically inspected. Currently, the white box approach doesn't fully solve the issue of IP traceability, which presents an enduring challenge for microprocessors and possibly other industries.

The RISC-V code is accessible to Thales and its clients and suppliers to fully inspect it. This capability is valuable for Thales because suppliers of proprietary solutions are reluctant to grant access to their code and when they do agree, the costs are high. The white box approach is critical for open source applications in the aerospace and defense sectors where security and safety are critical performance factors. For defense applications undertaken by Thales, clients and developers worry about security breaches such as a backdoor attack; a white box allows them to inspect the code. A Thales employee remarked, "In the telecom sector, with what happened with Huawei, we see that some suppliers from certain countries will not be allowed to deliver to other countries because we don't know what is inside their software and hardware. That's why the white box approach helps very much."

Also, in safety-critical industries like defense and avionics where Thales is active, certification authorities expect a fully mastered processor that qualifies for worst-case execution time, whereas most processors are designed to offer the maximum average performance. Worst-case execution time refers to the maximum length of time a computational task could take to execute. This parameter is essential for systems working in real time in defense equipment or airplanes where reliability is essential.

Another benefit of the white box approach is the transparency in terms of intellectual property. When using open source, a commercial firm can ensure it is not infringing upon another company's intellectual property. For Thales, avoiding intellectual property infringement is critical. However, since the intellectual property landscape for processors is 
complex, infringement remains an industry-wide challenge that will require new solutions to create and guarantee full transparency.

This adoption factor is particularly relevant for sectors and applications where security and safety are paramount. All interviewees agreed that open source provides full access and contributes to transparency. Nevertheless, our interviewees mentioned that for industries other than defense and aviation, security and safety concerns might not be as critical. For instance, even if the Internet of Things generates security concerns, the first security priority might not be having full transparency on an ISA.

\section{Factor 5: Ability to Select Suppliers Other Than Dominant Players}

Relying on a single supplier creates a risk of dependency that can leave companies vulnerable to price increases or with limited access to alternative solutions when the quality of technical support and services proves unsatisfactory. An H-ROSE can minimize such risks and offer flexibility because it provides access to multiple supply sources. An H-ROSE allows the development of a dual source approach that mitigates supply risks throughout a product's life cycle.

Flexibility is especially important for Thales because it has specific industry requirements. In the defense industry, export controls prevent a firm from selling its products to certain clients. Consequently, risk comes with having suppliers located in a country whose government decides to strengthen its export control regulations. Foreign acquisition of a supplier can lead to further export restrictions depending on the acquiring firm's home country. "One supplier used to be European, it was purchased by a Japanese company; tomorrow it can become a Chinese company. So, in 10 years from now, IP can be localized in a different country from now. With proprietary IP there is no way you can control this. With open source, you keep the control of this," said one Thales interviewee. Some observers (Glandien 2019) have suggested that unilateral trade sanctions are forcing China to favor the adoption of RISC-V. Interest in RISC-V is also strong in countries like India and Pakistan whose governments have officially adopted it as the national ISA.

For some defense applications, companies like Thales must provide technical redundancies that use different technologies or come from different suppliers. This requirement exists in case a system malfunctions; a second one can replace it.

Thales can also benefit from locating its supply chain in different countries in response to local sourcing requirements. For example, Thales can benefit from the RISC-V ecosystem in Madras, India, because of the company's solid market position there. Open source solutions help eliminate these risks and create benefits. Another industry-specific benefit of open source is that it offers the possibility to change suppliers with limited upfront investment. In industries with long product life cycles, if a sole supplier of hardware decides to stop selling a product, the client must buy a significant inventory to cover for maintenance, repair, and end-of-life management of its own products. Open source comes with the promise that companies can switch suppliers or even re-integrate some activities and keep control of its product over time. For Thales, such long-term benefits appear attractive, but they remain a long-term, uncertain bet. 
In weighing whether to join an H-ROSE, companies need to consider all benefits associated with flexibility in the choice of suppliers. In Thales' case, the industry-specific benefits described above go well beyond the possibility of improving the company's bargaining position with a dominant supplier.

\section{Factor 6: Permissive License Agreements}

Permissive license agreements are an essential factor for an HROSE's success as they allow companies to create proprietary derivative work out of open source solutions. However, permissive license agreements may reduce the incentives for some member firms to contribute back to the ecosystem, which can in turn prove detrimental to the ecosystem's development.

The RISC-V Foundation has chosen to provide access to its ISA through a Berkeley Software Distribution (BSD) license, which means users, under the terms of this specific license, have no obligation to make their code and the code of associated components available under the same license. This prevents what is called "contamination effects" on the proprietary components of the design (Table 4). Thales can decide freely if it will open source its own code or keep it proprietary. The RISC-V Foundation and specific projects initiated by some members offer diverse opportunities to contribute back to the ecosystem. Beyond the licenses, the RISC-V Foundation has established clear reciprocal obligations for members that contribute to the design of the core architecture. To become a foundation member, organizations must agree that they will not sue each other on the core instructions; if they do, the RISC-V Foundation will sanction them, and they will lose their right to use the core instructions.

\section{- - Table 4 near here - -}

Companies considering joining an H-ROSE must keep in mind that understanding the legal implications of the license used is not enough. With permissive license agreements, companies must also understand how members are expected to contribute back to the $\mathrm{H}$ ROSE. Specific mechanisms can help to channel resources and money either to a foundation or to some projects that support the H-ROSE. The perception of open source may be the significant outcome from the Thales case. Many companies' leaders have preconceived ideas of open source regarding disclosing their designs. We found there is a general lack of knowledge about the different types of licenses that can support open source initiatives and their respective business impacts. Licensing matters need to be better understood to favor the adoption of H-ROSE.

\section{Factor 7: A Growing and Active Ecosystem}

This adoption factor builds on three advantages that mutually reinforce each other: the development of a critical mass of adopters on the user side, the development of a critical mass of developers, and the governance rules of the open source community and ecosystem. Before deciding to adopt RISC-V, Thales needed evidence that other commercial firms were adopting and supporting it. 
The RISC-V Foundation's growing membership provides credibility and enhances the resources that support RISC-V. The Foundation's growing membership is only a starting point - active participation is even more important. Adoption of RISC-V by commercial firms from many sectors signals that the solution could become a dominant design in the future. The participation of technology firms like Google, Western Digital, NVIDIA, and Tesla is particularly relevant. Leading companies' contributions of RISC-V extensions, and sometimes full processors as open source, signals long-term investments are being made.

Some companies might maintain secrecy about their early adoption of RISC-V to protect their relationships with their proprietary solutions providers (Yoshida 2018). During an interview in 2019, Ted Marena, senior director of the RISC-V ecosystem at Western Digital, described a specific organization initiated by Western Digital and other companies to support the development of the RISC-V ecosystem. "It's an open hardware development group; the idea is to collaboratively work on hardware so everybody can benefit from this. We are going to use as many open source tools as possible. Some proprietary IP will remain. But as much as possible, we will use open source tools. There's big benefit for all in this," Marena said.

Having a critical mass of developers is key. Commercial firms need to access complementary IP together with design, testing, and production capabilities that support RISC-V implementation. They need to hire developers that are knowledgeable about RISC-V, and they need access to other companies that can assist with design support, design tools, production, and other technical aspects. The RISC-V ecosystem is developing quickly with initiatives in the US, India, and China. Nonetheless, fragmentation is an inherent risk in such rapid development. In the future, the RISC-V ecosystem needs to maintain a common standard to ensure the quality of the solution. The RISC-V Foundation actively promotes use of a single repository and a compliance verification tool. Its rules of governance support the development of a growing and active ecosystem. The Foundation has a multi-tier membership with a limited number of platinum members that can influence the standard. Other members of the RISC-V ecosystem can support the RISC-V Foundation and its associated projects; they can also contribute RISC-V extensions and share their industry knowledge and experience with other ecosystem members.

The RISC-V Foundation protects the ISA using a trademark and guarantees compatibility within the development community. It also holds events to promote the ISA. The fact that some respected experts from public institutes, universities, and standards bodies are foundation members is a positive sign for commercial firms. The RISC-V Foundation is creating certification schemes for individuals working with RISC-V and compliance tools so users can claim their processor is a RISC-V processor.

To conclude, having a growing and active ecosystem is a key adoption factor. If the ecosystem grows too quickly, fragmentation may occur that could lead to mistrust of the ISA's integrity. If the ecosystem grows too slowly, its success could be jeopardized. Companies assessing an H-ROSE as an option need to explore beyond a list of company names. In an active H-ROSE, it is easy to meet existing members and gather information about activities and projects across the ecosystem. To ensure they can cover all their 
needs and maintain flexibility, companies should also assess the dynamism of the ecosystem in different regions.

\section{Adoption Factors and Participation in Hardware-Rich Open Source Ecosystems}

We mapped the influence of the factors to determine which factors act as foundations and which ones result from others (Figure 1). We determined that the technical solution's stability and modularity (Factor 3), the use of permissive licenses (Factor 6), and the development of a growing and active ecosystem (Factor 7) are foundation factors. The three foundation factors enable other factors - namely, the white box approach, the flexibility of the design process, and the possibility of using suppliers outside of dominant players. Cost impacts only appear as an outcome allowed by other factors. Our analysis was confirmed by Rick O'Connor, current president and CEO of the OpenHW Group and former executive director of the RISC-V Foundation. He suggested three reasons why previous open source initiative have failed for microprocessors: the quality of the IP, an ecosystem offering a long-term roadmap, and legal issues that usually scare legal teams in large companies. These three reasons correspond with the three foundation factors we identified.

\section{- - Figure 1 near here - -}

\section{H-ROSE Adoption Factors vs. Open Source Software}

We compared the seven adoption factors with the results of open source software adoption studies conducted by Morgan and Finnegan (2007) and Dedrick and West (2003). While the seven factors appeared to be common to the two types of ecosytems, we identified a few specificities for H-ROSEs. We determined that quality, validation, and supply chain management are more stringent for an H-ROSE than for open source software because multiple software and hardware components need to be combined. Accordingly, a well-coordinated H-ROSE needs resources and capabilities to establish and apply compliance, validation, and certification procedures. In 2015, there was public concern about the certification procedures of the Open Compute Project, an H-ROSE focused on data centers (Judge 2015). These concerns have not prevented further development of the ecosystem, but H-ROSEs must manage such issues effectively. Within an H-ROSE, contradictory forces such as ecosystem growth and the need for stability and reliability require active management.

H-ROSEs require heterogenous ecosystems that match diverse requirements with a broad set of capabilities and actors. For instance, Apollo Auto has attracted 12,000 developers (Wiggers 2019). A large membership can make an ecosystem attractive as it offers unique and diverse collaboration opportunities amongst heterogenous actors. However, specific needs in terms of functionalities, quality, or volumes might be difficult to fulfil rapidly and could lead to mistrust within the ecosystem and a loss of competitive position. In the telecom sector, OpenRan (TIP) is scaled up progressively (Morris 2019) to address obstacles to the development of a fully effective supply chain. 
While companies like Microsoft, Facebook, and Google may consider open source the new normal, in other industries, perceptions about appropriatentess, concerns about licensing constraints, and questions about business model viability may yet deter companies from adopting an H-ROSE. Companies need more education on the benefits and risks associated with the adoption of open source solutions, especially in the hardware sector.

\section{Reflecting on Thales' Experience with RISC-V}

Following the study undertaken within Thales in 2018, the company determined RISC-V was a promising avenue for its future developments. Thales became a member of the RISC-V Foundation, began using RISC-V to develop critical embedded systems, and became active in the ecosystem. In collaboration with the RISC-V Foundation, Thales supports initiatives that promote and advance the use of RISC-V, including event organization, online events, meetups, etc. Thales is also taking part in several collaborations, including a partnership with the Indian Institute of Technology in Madras (IITM) to design a multi-core RISC-V processor for safety-critical domains like avionics - this open source design is available on GitLab. Thales is also a founding member of the OpenHW Group that aims to provide industrial grade RISC-V processors. This initiative builds on the Pulp family RISC-V processor designed by ETH-Zurich and includes collaboration with companies such as NXP and Alibaba.

The seven adoption factors, the analysis undertaken, and the conclusions reached remain relevant and valid. The European part of the RISC-V ecosystem, however, is less developed than anticipated - while this fact does not invalidate the seven adoption factors identified, it highlights the need for attention to regional aspects. Thales' experience continues to confirm that a strong and solid engagement is needed to derive benefits from an H-ROSE. A Thales technical director stressed that "Being a follower make no sense, and even if RISC-V is not our core business, Thales has had to become an active actor to really take advantage of open source hardware."

Companies that join an open source initiative need to adapt their culture. The Thales technical director noted, "Open source requires a different mindset. From a business perspective, it implies a departure from thinking close source and then deciding if we have something to share, we need to think openly by default and then decide what we need to keep internally. It is a cultural change that needs to be addressed." This need to address culture changes goes beyond adoption factors, but it reinforces the need to eliminate preconceived ideas and suggests that as H-ROSEs develop, companies will need to consider such culture changes.

\section{Conclusion}

Almost 20 years ago, Microsoft CEO Steve Balmer opposed open source software. Today, Microsoft, Facebook, Google, and others are building competitive advantages on top of open source cornerstones. Over the past 10 years the benefits of open source have been tested beyond software and a few H-ROSEs have emerged. Although it is too early to judge how successful H-ROSEs will ultimately be, the development of more H-ROSEs 
are likely. Our seven adoption factors can help firms decide whether joining an H-ROSE makes good business sense. Further investigation is needed, particularly regarding how to manage tensions between growth and stability within an H-ROSE. Our outcomes from the Thales case show that companies with limited exposure to open source inititiaves must consider significant culture changes as they start adopting an H-ROSE.

\section{References}

Adner, R. 2017. Ecosystem as Structure: An Actionable Construct for Strategy. Journal of Management 43(1), 39-58.

Bonvoisin, J., Mies, R., Boujut, J. F., and Stark, R. 2017. What is the 'source' of open source hardware? Journal of Open Hardware 1(1): 5.

Dedrick, J., and West, J. 2003. Why firms adopt open source platforms: A grounded theory of Innovation and standards adoption. In Proceedings of the Workshop on Standard Making: A Critical Research Frontier for Information Sys-Tems, pp. 236-57.

Dubois, C. 2017. Engineer spotlight: Shafy Eltoukhy, Head of SiFive's designshare program. All About Circuits, December 21. https://www.allaboutcircuits.com/news/engineer-spotlight-head-SifFves-Designshare-program-shafy-eltoukhy/

Eisenhardt, K. M. 1989. Building theories from case study research. The Academy of Management Review 14(4): 532-50.

Gambardella, A., and von Hippel, E. 2019. Open sourcing as a profit-maximizing strategy for downstream firms." Strategy Science 4(1): 41-57.

Glandien, A. 2019. A new blueprint for microprocessors challenges the industry's giants. The Economist, October 3. https://www.economist.com/science-and-technology/2019/10/03/a-new-blueprint-for-microprocessors-challenges-the-industrys-giants

Jacobides, M. G., Cennamo, C., and Gawer, A. 2018. Towards a theory of ecosystems. Strategic Management Journal 39(8): 2255-76.

Judge, P. 2015. OCP testing regime comes under fire. Data Center Dynamics, July 8. https://www.datacenterdynamics.com/news/ocp-testing-regime-comes-under-fire/.

Lerner, J., and Tirole, J. 2005. The economics of technology sharing: Open source and beyond. Journal of Economic Perspectives 19(2): 99-120.

Mariotto, F.L.,; Zanni, P. P., and De Moraes, G. 2014. What is the use of a single-case study in management research?. Rev. adm. empres 54(4): 358-369.

McKeefry, H. 2019. How open source hardware empowers procurement. EPS News (blog), February 19. https://epsnews.com/2019/02/19/open-source-empowersprocurement/ 
Morgan, L., and Finnegan, P. 2007. Benefits and drawbacks of open source software: An exploratory study of secondary software firms." In Open Source Development, Adoption and Innovation, edited by Joseph Feller, Brian Fitzgerald, Walt Scacchi, and Alberto Sillitti, 307-12. New York, NY: Springer US.

Morris, I. 2019. Facebook, Telefonica take OpenRAN 'live' in Peru, boast 450K subs. Light Reading, November 13. https://www.lightreading.com/facebook-telefonicatake-openran-live-in-peru-boast-450k-subs/d/d-id/755596.

Kauttu, P., and Rosado Murillo, L. F. 2017. Open hardware as an experimental innovation platform: Preliminary research questions and findings." CERN IdeaSquare Journal of Experimental Innovation 1(1): 26.

Nervos, X, X. 2019. Introduction to the RISC-V instruction set CKB-VM and its requirements-industry articles. All About Circuits, March 5. https://www.allaboutcircuits.com/industry-articles/introduction-to-the-risc-v-instruction-set-ckd-vm-and-itsrequirements/.

Peng, T. 2019. Alibaba is open-sourcing its powerful new RISC-V processor for 5G and AI. synced, July 25. https://medium.com/syncedreview/alibaba-is-open-sourcing-itspowerful-new-risc-v-processor-for-5g-and-ai-dcb6f4eebbc4

Pénin, J. 2011. Open source innovation: Towards a generalization of the open source model beyond software. Revue d'économie Industrielle 136: 65-88.

Tsoukas, H. 2009. Craving for generality and small-n studies: The Wittgesteinian approach towards the epistemology of the particular in organization and management studies." In The SAGE Handbook of Organizational Research Methods, edited by David Buchanan and Alan Bryman. London, UK: Sage, pp. 358-369.

Tusinschi, N. 2019. A holistic view of RISC-V verification. Semiconductor Engineering (blog), August 7. https://semiengineering.com/a-holistic-view-of-risc-v-verification/

Wejnert, B. 2002. Integrating models of diffusion of innovations: A conceptual framework. Annual Review of Sociology 28(1): 297-326.

Wiggers, K. 2019. Baidu releases Apollo 5.0 with upgrades for 'complex' road scenarios." VentureBeat (blog), July 1. https://venturebeat.com/2019/07/01/baidu-releasesapollo-5-0-with-upgrades-for-complex-road-scenarios/

Yoshida, J. 2018. There's more to the RISC-V China story. EETimes, March 11. https://www.eetimes.com/author.asp?section_id=36\&doc_id=1334001 
Table 1.-Five H-ROSE initiatives

\begin{tabular}{|c|c|c|c|c|}
\hline Name & System & $\begin{array}{l}\text { Some Ecosystem } \\
\text { Members }\end{array}$ & $\begin{array}{l}\text { What Is Open } \\
\text { Source? }\end{array}$ & Comments \\
\hline RISC-V & $\begin{array}{l}\text { Focus on the } \\
\text { instruction set } \\
\text { architecture } \\
\text { that is inte- } \\
\text { grated within a } \\
\text { processor. }\end{array}$ & $\begin{array}{l}\text { 400+ members, in- } \\
\text { cluding Western } \\
\text { Digital, NVDIA, } \\
\text { Google, Alibaba, } \\
\text { NXP, Samsung, } \\
\text { Qualcomm }\end{array}$ & $\begin{array}{l}\text { The instruction set } \\
\text { architecture }\end{array}$ & $\begin{array}{l}\text { The RISC-V Foundation } \\
\text { was established in } 2015 . \\
\text { Today, complementary } \\
\text { initiatives exist to } \\
\text { broaden openness and } \\
\text { offer some processor as } \\
\text { open hardware. }\end{array}$ \\
\hline $\begin{array}{l}\text { Open Compute } \\
\text { Project (OCP) }\end{array}$ & $\begin{array}{l}\text { Hardware and } \\
\text { software for } \\
\text { data center in- } \\
\text { frastructures }\end{array}$ & $\begin{array}{l}178 \text { members in- } \\
\text { cluding Facebook, } \\
\text { Goldman Sachs, } \\
\text { IBM, Rackspace, } \\
\text { HP, Microsoft, } \\
\text { Nokia, Huawei }\end{array}$ & $\begin{array}{l}\text { Hardware and soft- } \\
\text { ware components }\end{array}$ & $\begin{array}{l}\text { The OCP was estab- } \\
\text { lished in 2011. Some } \\
\text { software projects have } \\
\text { been recently imitated to } \\
\text { complement hardware } \\
\text { ones. }\end{array}$ \\
\hline $\begin{array}{l}\text { Telecom } \\
\text { Infra Project } \\
\text { (TIP) }\end{array}$ & $\begin{array}{l}\text { Telecom net- } \\
\text { work infra- } \\
\text { structure }\end{array}$ & $\begin{array}{l}\text { 500+ members, in- } \\
\text { cluding Deutsche } \\
\text { Telekom, Face- } \\
\text { book, Telefonica, } \\
\text { Intel, Nokia, Voda- } \\
\text { fone, BT }\end{array}$ & $\begin{array}{l}\text { Hardware and soft- } \\
\text { ware components }\end{array}$ & $\begin{array}{l}\text { TIP was established in } \\
2016 . \text { It has projects, } \\
\text { lab, and startup acceler- } \\
\text { ators. }\end{array}$ \\
\hline Apollo Auto & $\begin{array}{l}\text { Autonomous } \\
\text { driving plat- } \\
\text { form }\end{array}$ & $\begin{array}{l}\text { 130+ members, in- } \\
\text { cluding Baidu, } \\
\text { BMW, BOSCH, } \\
\text { Continental, Flex, } \\
\text { Ford, PSA, In- } \\
\text { fineon, Intel, Mi- } \\
\text { crosoft, Nvidia, } \\
\text { NXP, TomTom }\end{array}$ & Software & $\begin{array}{l}\text { Baidu initiated this eco- } \\
\text { system in } 2017 \text { to sup- } \\
\text { port its strategy to be- } \\
\text { come a key player in au- } \\
\text { tonomous driving. }\end{array}$ \\
\hline
\end{tabular}




\begin{tabular}{|l|l|l|l|l|}
\hline $\begin{array}{l}\text { Lora } \\
\text { Alliance }\end{array}$ & $\begin{array}{l}\text { IoT open } \\
\text { standard }\end{array}$ & $\begin{array}{l}\text { 500+ members in- } \\
\text { cluding Actility, } \\
\text { Cisco, Orange, } \\
\text { Alibaba, Comcast, } \\
\text { Bouygues Telecom, } \\
\text { Semtech, Tencent, } \\
\text { STMicroelectronics }\end{array}$ & $\begin{array}{l}\text { Some software and } \\
\text { hardware compo- } \\
\text { nents }\end{array}$ & $\begin{array}{l}\text { This ecosystem was ini- } \\
\text { tiated in 2015. Beyond } \\
\text { the Lorawan Protocol, } \\
\text { available as an open } \\
\text { standard, more open } \\
\text { source components have } \\
\text { been made available } \\
\text { over time. }\end{array}$ \\
& & & & \\
\hline
\end{tabular}


Table 2.-Interviewees

\begin{tabular}{|l|l|l|}
\hline Role & Company & Ecosystem \\
\hline Sub-Contract Manager & Thales & RISC-V \\
\hline Procurement Director & Thales & RISC-V \\
\hline $\begin{array}{l}\text { Embedded IoT \& Compu- } \\
\text { ting R\&T Manager }\end{array}$ & Thales & RISC-V \\
\hline $\begin{array}{l}\text { VP Research and Technol- } \\
\text { ogy Hardware }\end{array}$ & Thales & RISC-V \\
\hline $\begin{array}{l}\text { Technology and Innova- } \\
\text { tion Manager }\end{array}$ & Thales & RISC-V \\
\hline VP Marketing \& Alliances & SYSGO / Thales & RISC-V \\
\hline Engineering Manager & SYSGO / Thales & RISC-V \\
\hline $\begin{array}{l}\text { Business Development, } \\
\text { Board Member }\end{array}$ & ANTMICRO & RISC-V \\
\hline Executive Director & RISC-V Foundation & RISC-V \\
\hline $\begin{array}{l}\text { Director RISC-V Ecosys- } \\
\text { tem }\end{array}$ & $\begin{array}{l}\text { WESTERN DIGITAL } \\
\text { RISC-V Foundation }\end{array}$ & RISC-V \\
\hline $\begin{array}{l}\text { Vice President } \\
\text { Channel Development }\end{array}$ & Open Compute Project & OCP \\
\hline Software Engineer & Developer & LORA \\
\hline $\begin{array}{l}\text { Leader of the CERN Open } \\
\text { Hardware community }\end{array}$ & CERN & CERN White Rabbit \\
\hline Procurement Officer & CERN & CERN White Rabbit \\
\hline
\end{tabular}


Table 3.-Adoption factors

\begin{tabular}{|c|c|}
\hline Factor & Sub-Factors \\
\hline $\begin{array}{l}\text { Factor 1: Total Cost Opti- } \\
\text { misation, Including the } \\
\text { Cost of Accessing Innova- } \\
\text { tion }\end{array}$ & $\begin{array}{l}\text { Adoption advantages: } \\
-\quad \text { Lower intellectual property cost } \\
-\quad \text { Lower design cost through reuse of open source building } \\
\text { blocks } \\
-\quad \text { Pooling of creativity and resources needed to innovate } \\
\text { Limitations: } \\
-\quad \text { Advantages can be limited to specific application } \\
\end{array}$ \\
\hline $\begin{array}{l}\text { Factor 2: Flexible and } \\
\text { Rapid Design Process }\end{array}$ & $\begin{array}{l}\text { Adoption advantages: } \\
-\quad \text { Reduction of administrative steps and upstream innovation } \\
\text { barriers } \\
-\quad \text { Possibility of accessing a broad set of rapid design and proto- } \\
\text { typing capabilities } \\
\text { Limitations: } \\
-\quad \text { Advantages are more specific to custom-purposed processors } \\
-\quad \text { Industry leaders have started to adapt their offerings }\end{array}$ \\
\hline $\begin{array}{l}\text { Factor 3: Stability and } \\
\text { Modularity }\end{array}$ & $\begin{array}{l}\text { Adoption advantages: } \\
-\quad \text { Stability of the solution } \\
-\quad \text { Modularity of the solution } \\
\text { Limitations: } \\
-\quad \text { Lack of complete verification tools }\end{array}$ \\
\hline $\begin{array}{l}\text { Factor 4: The White Box } \\
\text { Approach }\end{array}$ & $\begin{array}{l}\text { Adoption advantages: } \\
\text { - } \quad \text { Ability to inspect the content of the solution (software, hard- } \\
\text { ware, IP) is key for safety and security* } \\
\text { - } \quad \text { Transparency in terms of intellectual property prevents poten- } \\
\text { tial IP infringements } \\
\text { Limitations: } \\
\text { - Traceability of IP is difficult to ensure but this is an industry- } \\
\text { wide challenge }\end{array}$ \\
\hline $\begin{array}{l}\text { Factor 5: Possibility to Se- } \\
\text { lect Suppliers Outside of } \\
\text { Dominant Players }\end{array}$ & $\begin{array}{l}\text { Adoption advantages: } \\
-\quad \text { Ability to develop a dual source approach to mitigate supply } \\
\text { risks } \\
-\quad \text { Provides flexibility for maintenance and repair* } \\
-\quad \text { Provides flexibility for end-of-life issues* } \\
\text { Limitations: } \\
-\quad \text { Some long-term advantages in a hard-to-predict context }\end{array}$ \\
\hline $\begin{array}{l}\text { Factor 6: Permissive Li- } \\
\text { cense Agreements }\end{array}$ & $\begin{array}{l}\text { Adoption advantages: } \\
-\quad \text { Possibility to create proprietary derivative solutions } \\
\text { Limitations: } \\
-\quad \text { Fear that open source licenses will oblige firms to disclose } \\
\text { their design } \\
-\quad \text { Less incentives to contribute back to the ecosystem }\end{array}$ \\
\hline $\begin{array}{l}\text { Factor 7: A Growing and } \\
\text { Active Ecosystem }\end{array}$ & $\begin{array}{l}\text { Adoption advantages: } \\
-\quad \text { Development of a critical mass of adopters on the user side }\end{array}$ \\
\hline
\end{tabular}




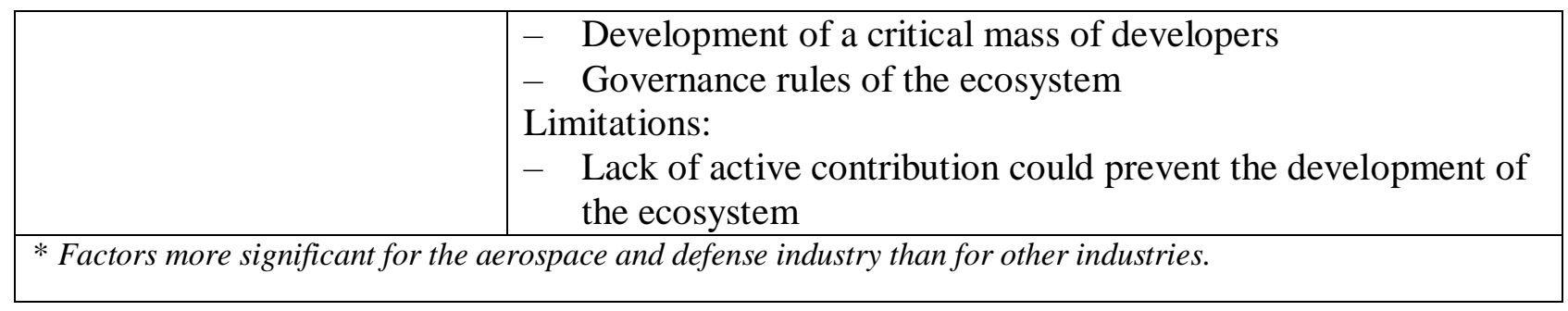

Table 4.-Open source licenses

\begin{tabular}{|c|c|c|c|}
\hline Type of License & $\begin{array}{l}\text { Copyleft License With } \\
\text { Contaminating Effect }\end{array}$ & $\begin{array}{l}\text { Copyleft License } \\
\text { Without Contaminat- } \\
\text { ing Effect }\end{array}$ & $\begin{array}{l}\text { Permissive } \\
\text { License }\end{array}$ \\
\hline Characteristics & $\begin{array}{l}\text { Oblige the redistribu- } \\
\text { tion of the code and as- } \\
\text { sociated components } \\
\text { under the same license. } \\
\text { These obligations im- } \\
\text { pacts on proprietary } \\
\text { parts and creates con- } \\
\text { tamination. }\end{array}$ & $\begin{array}{l}\text { Oblige the redistribu- } \\
\text { tion of the code under } \\
\text { the same license. } \\
\text { Combination with pro- } \\
\text { prietary code is possi- } \\
\text { ble without contamina- } \\
\text { tion. }\end{array}$ & $\begin{array}{l}\text { Permit modification and } \\
\text { distribution in compliance } \\
\text { with the license obliga- } \\
\text { tions. } \\
\text { Permit the creation of pro- } \\
\text { prietary derivative work } \\
\text { without having to make the } \\
\text { code available. }\end{array}$ \\
\hline $\begin{array}{l}\text { Example of Li- } \\
\text { censes }\end{array}$ & $\begin{array}{l}\text { GPL V2 } \\
\text { GPL V3 } \\
\text { AGPL V3 }\end{array}$ & $\begin{array}{l}\text { EPL V1.0 } \\
\text { LGPL V2 } \\
\text { Mozilla PL V1.1 }\end{array}$ & $\begin{array}{l}\text { MIT } \\
\text { Apache V2 } \\
\text { BDS }\end{array}$ \\
\hline
\end{tabular}


Figure captions:

Figure 1.-Impact across factors 